\title{
Editorial
}

\section{Grammatik im DaF-Unterricht}

\author{
Gabriela Perrig/Klaus Peter/Michael Schümann (Bern)
}

Das Verhältnis von linguistischer und didaktischer Grammatik im DaF-Unterricht wird schon seit Jahrzehnten kontrovers diskutiert. Im besten Falle bereichern beide einander, im schlechtesten Fall kapselt man sich voneinander ab. Die Grundpositionen sind klar: Die didaktische Seite hält eine massive Komplexitätsreduktion der grammatischen Beschreibung für geboten, Maßstab ist die kognitive Verarbeitbarkeit bei den Lernenden. Auf der anderen Seite steht die Sprachwissenschaft, die Präzision und deskriptive Adäquatheit anstrebt. Aus der Sicht der Lernenden ist beides unbefriedigend: sowohl eine so komplizierte Darstellung, dass sie nicht memorierbar oder praktisch anwendbar ist, als auch eine zu starke Vereinfachung der Regeldarstellung, die zu falschen Ergebnissen führt.

Dieses Spannungsverhältnis von wissenschaftlicher Theorie einerseits und deren Anwendung in der Praxis andererseits, das sich u. a. bereits in mehreren Themenheften von Linguistik online niedergeschlagen hat (Linguistik online 9/2001, 10/2002, 41/2010), war Gegenstand eines Workshops, der im Rahmen der 4. Tagung "Deutsche Sprachwissenschaft in Italien" im Februar 2010 abgehalten wurde. Ziel des Workshops war, anhand ausgewählter Grammatikprobleme zu diskutieren, wie sprachliche Phänomene linguistisch adäquat und zugleich in Hinblick auf die Praxis im Fremdsprachenunterricht sinnvoll beschrieben werden können.

Die in diesem Band versammelten sechs Beiträge aus dem Workshop beschäftigen sich mit insgesamt vier unterschiedlichen Themen, von denen zwei davon - Genus und Tempus - gewissermaßen zu den Klassikern im DaF-Unterricht zählen, während die anderen beiden Themen - kausale Verknüpfungsmittel und Interrogationen - zu Unrecht eine oft untergeordnete Rolle spielen. Alle Autorinnen kennen die Probleme der praktischen Vermittlung aus eigener Erfahrung.

Den Auftakt machen zwei Beiträge zum Thema Genus:

Marie Antoinette Rieger beschäftigt sich in ihrem Aufsatz "Genus im DaF-Unterricht in Italien: Was machen die Lerner?" im Rahmen einer empirischen Untersuchung mit der Frage, inwiefern die Genuszuweisung im Deutschen für italienischsprachige Lernende überhaupt ein Lernproblem darstellt und an welchen sprachlichen Kriterien sich die Lernenden bei der $\mathrm{Zu}$ weisung von Genus orientieren.

Marion Weerning greift in ihrem Artikel "Genus im DaF-Unterricht in Italien: Was sagen Lehrwerke und Grammatiken" komplementär Fragen der didaktischen Vermittlung des Themas Genus auf. Sie untersucht an italienischen Schulen und Universitäten gängige Lehrwerke und didaktische Grammatiken, um zu ermitteln, wie Lernenden in den Lehrwerken die Kategorie Genus nähergebracht wird, was sie dort über das Genus und seine Funktionen sowie über Kriterien der Genuszuweisung erfahren, und schließlich, wie Lehrwerke die Lernenden dabei zu unterstützen versuchen, das richtige Genus mental zu speichern.

$\mathrm{Zu}$ einem Themenkomplex - der Distribution von Präteritum und Perfekt und einer DaFgerechten Umsetzung dieses Lerngegenstands - gab es gleich mehrere Beiträge, die im Workshop in Form eines Panels diskutiert wurden. Zwei der Beiträge sind in das vorliegende Themenheft eingegangen: 
Korakoch Attaviriyanupap wirft unter der Überschrift "Der Gebrauch des Perfekts - Ein Erklärungsmodell aus thailändischer Perspektive" einen Blick auf die Lernschwierigkeiten speziell thailändischer Lerner beim Tempuserwerb. Sie spricht sich gegen zu große Vereinfachungen bei der didaktischen Regelformulierung aus, etwa indem pauschal Präteritum vs. Perfekt mit geschriebener vs. gesprochener Sprache verbunden werden. In ihrem eigenen Modell setzt Attaviriyanupap das Merkmal Abgeschlossenheit an, es verbindet Tempus und Aspekt - für Sprachen wie das Thailändische, welche zwar kein morphologisches Tempus, jedoch Aspektmarker besitzen, kann auf dieser Parallele aufgebaut werden. Ebenso sollte auf die Kontrastierung mit dem Englischen (simple past vs. present perfect) Wert gelegt werden im Sinne eines DaFnE-Ansatzes ('Deutsch als Fremdsprache nach Englisch'), der die Ressourcen der ersten Fremdsprache für den nachfolgenden Fremdspracherwerb nutzen möchte.

Nicole Schumacher geht in ihrem Aufsatz "Nachzustand, Distanz und Aspektualität als Komponenten einer formfokussierten Steuerung von Perfekt und Präteritum" ebenfalls von einer aspektuellen Komponente bei der Bedeutungsbeschreibung von Präteritum und Perfekt aus. Sie arbeitet mit den Konzepten Nachzustand und Distanz, um die Distribution der Tempora zu erfassen. Besonderes Augenmerk legt sie darauf, die Lerner auf FormBedeutungszusammenhänge im Sinne einer formfokussierten Steuerung aufmerksam zu machen. Sie diskutiert schließlich eine Reihe von Möglichkeiten, wie eine solche Formfokussierung in den Fremdsprachenunterricht eingebunden werden kann.

Die letzten beiden Aufsätze des Hefts widmen sich schließlich der Beschreibung und Vermittlung von syntaktischen Phänomenen:

Sabrina Ballestracci erstellt in ihrem Aufsatz "Die kausalen Verknüpfungsmittel des Deutschen und des Italienischen. Eine kontrastive Beschreibung unter formalem und funktionalem Aspekt" auf der Grundlage von fünf deutschen und vier italienischen Grammatiken ein Inventar der kausalen Verknüpfungsmittel beider Sprachen. Sie geht dabei von einem weiten, zunächst semantisch definierten Kausalitätsbegriff aus. Auf die ausführliche Beschreibung der strukturellen Merkmale der kausalen Verknüpfungsmittel folgt eine Zusammenfassung der Ähnlichkeiten und Unterschiede, welche sich als Grundlage für eine kontrastive didaktische Darstellung der deutschen Strukturen für italienische Lernende anbietet.

Martina Rost-Roth weist in ihrem Beitrag "Formen und Funktionen von Interrogationen. Fragen in grammatischen Beschreibungen, empirischen Befunden und Lehrwerken für Deutsch als Fremdsprache" nach, dass die Beschreibungen von Interrogationen in Grammatiken den empirischen Befunden zu Fragesätzen oft nicht gerecht werden; ein ähnliches Bild zeigt sich in DaF-Lehrwerken. Dies ist für DaF-Lernende deshalb besonders misslich, weil für sie z. B. die Fähigkeit zur Formulierung von Nachfragen zur Verständnissicherung hochrelevant ist. Die Autorin plädiert deshalb dafür, Interrogationen im DaF-Unterricht umfassender und auf der Grundlage von empirischen Untersuchungen systematischer und verständlicher zu vermitteln. 